\title{
Circle homeomorphisms with flat critical points
}

by

Grzegorz Świątek (Stony Brook, N.Y.)*

Abstract. We prove that for a certain class of degree one circle homeomorphisms all orbits are dense provided the rotation number is irrational. This class includes some maps with flat critical points.

\section{Introduction}

1.1. General remarks. A classical theorem of Denjoy states that a circle diffeomorphism of degree one which satisfies certain mild smoothness conditions has dense orbits whenever its rotation number is irrational. The question whether the same is true for analytic homeomorphisms for which the derivative may turn into 0 was answered affirmatively by Yoccoz [3] only a few years ago. The proof used a new technique which allowed some control of the way in which the function distorted proportions of adjacent intervals. Subsequently, a technique based on a very similar principle was developed in [2]. We are going to use it as a main tool in the present work.

The technique works well for some kinds of critical points, like those that occur in analytic mappings. However, there are singularities for which the method breaks down. They are often called "flat critical points" and the simplest example in this class is the singularity of the type of

$$
\operatorname{sgn}(x) \exp (-1 /|x|) \quad \text { at } 0 .
$$

The purpose of this paper is to extend Yoccoz's result to maps with a certain type of flat critical point. The class of singularities that we allow is not very large; in fact, it could not be too general as there are examples of $C^{\infty}$ homeomorphisms with flat critical points whose orbits are not dense (see [1]).

We demand that the maps in our class have only one flat singularity, that they are suitably regular in the proximity of the singularity, and the most restrictive assumption we make is that locally they look the same on both sides of the flat critical point.

* This work was originated while the author was with the Institute of Mathematics of the Polish Academy of Sciences in Warsaw and concluded at the Institute for Advanced Study in Princeton. 
What is interesting is that we do not have to impose restrictions on "flatness" of the singularity $\left({ }^{1}\right)$. Hence, it seems that it is not "flatness" that makes the Denjoy theorem break down. In fact, this paper was originated by efforts to find a "Denjoy counterexample" that could be given by a simple explicit formula. Our result does not prove it is impossible but merely shows that we still do not understand the nature of flat critical points well. Hopefully, some of the techniques we developed will be useful in future research.

On the positive side, our work shows examples of circle homeomorphisms with an irrational rotation number of constant type which are topologically conjugate to the rotation but the conjugacy is not quasisymmetric.

\subsection{The cross-ratio inequality}

Definition 1.1. Let $a, b, c, d$ be points of the line. We will denote quadruples of points with script capital letters, so let us call this one $\mathscr{A}$. The cross-ratio of $\mathscr{A}$ is defined by

$$
\operatorname{Cr}(\mathscr{A}):=\frac{(b-a) \cdot(d-c)}{(c-a) \cdot(d-b)} .
$$

Definition 1.2. Furthermore, let us consider a map $h$ which is strictly monotone on the interval $(a, d)$. The distortion of the cross-ratio under $h$ is defined to be

$$
D(\mathscr{A} ; h):=\frac{\operatorname{Cr}(h(\mathscr{A}))}{\operatorname{Cr}(\mathscr{A})} .
$$

Next, we view the real line as a branched cover of some manifold $M$ (in our case, $M$ is the circle and the cover is a regular, not branched, cover). Let $\pi$ denote the covering map.

DEFINITION 1.3. A monotone map $h$ is said to satisfy the cross-ratio inequality if it is possible to pick a sequence of constants $C_{k}$ so as to satisfy the following property for any sequence $\mathscr{A}_{i}=\left(a_{i}<b_{i}<c_{i}<d_{i}\right)$ where $i$ ranges from 0 to some arbitrary $n$ :

If

- for each $i=0, \ldots, n$ the map $h$ is defined and strictly monotone on $\left[a_{i}, d_{i}\right]$,

- no point of $M$ belongs to more than $k$ images of $a_{i}, d_{i}$ by $\pi$ for various $i$, then

$$
\prod_{i=0}^{n} D\left(\mathscr{A}_{i} ; h\right) \leqslant C_{k}
$$

Remarks on the cross-ratio inequality. The strength of the cross-ratio inequality is in the fact that the constants $C_{k}$ do not depend on the choice of the sequence $\mathscr{A}_{i}$ and, in particular, are independent of $n$. Note also that $h$ does not have to be defined on the
whole line.

$\left.{ }^{1}\right)$ By which we mean how fast the derivative tends to 0.
To verify the cross-ratio inequality is usually tiresome. It was done in [2]. We also mention a criterion which was communicated to the author by M. Herman:

THE CRITERION. If $M$ is a compact manifold and $h$ is quasisymmetric and also the lift of a map defined on $M$; and if it is possible to find finite sequences of open sets $U_{j}$ on which $\pi$ can be inverted and points $u_{j} \in U_{j}$ so that the following is satisfied:

- the function $h$ restricted to $R \backslash \bigcup \pi^{-1}\left(U_{j}\right)$ is $C^{1,1}$ and its derivative is bounded away from 0 ,

- if $a<b<c<d$ belong to a connected component of $\pi^{-1}\left(U_{j}\right)-\pi^{-1}\left(u_{j}\right)$ for some $j$, then $D(a, b, c, d ; h) \leqslant 1$,

then $h$ satisfies the cross-ratio inequality.

1.3. Formal statement of the results

Terminological remarks. The circle is the unit circle in the complex plane covered by the real line in the standard way: $x \rightarrow \exp (2 \pi i x)$.

A map $F$ from the line to itself projects to the circle if and only if it satisfies $F(x+1)-F(x) \in Z$ for all $x$. This difference does not depend on $x$ and is equal to the topological degree of the circle map. The map $F$ is called the lift of the circle map.

We do not see a need to make a clear distinction between objects on the line and the corresponding objects on the circle. Formally, we will use notations that make sense on the real line, but we will freely speak of the underlying objects as living on the circle. If we want to emphasize that a formula concerns objects on the circle, we will write $(\bmod 1)$ next to it.

HyPOTHESES. The map $h$ is the lift of a degree one circle homeomorphism with an irrational rotation number. The map is at least once continuously differentiable.

Furthermore, there is one distinguished point on the circle, identified with 0 , and $h$ restricted to $\boldsymbol{R} \backslash \boldsymbol{Z}$ satisfies the cross-ratio inequality.

On a set $U$, which is an open neighborhood of 0 , the map $h$ has special properties. It does not increase any cross-ratio $\operatorname{Cr}\left(a_{3,}, c, d\right)$ provided that $a<b<c<d$ and the interval $(a, d)$ is contained in $U$ but does not include 0. Moreover, $h$ on $U$ is assumed to be expressible in the form

$$
h(x)=\operatorname{sgn}(x) \exp (-f(|x|))+h(0) .
$$

The map $f: \boldsymbol{R}_{+} \rightarrow \boldsymbol{R}_{+}$satisfies the following list of technical requirements:

$$
\begin{aligned}
& \text { 1) } f^{\prime}<0 \\
& \text { 2) } f^{\prime \prime}>0 \\
& \text { 3) } \lim _{x \rightarrow 0} f^{\prime \prime}(x) /\left(f^{\prime}\right)^{2}(2 x)=0, \\
& \text { 4) for every positive } \alpha
\end{aligned}
$$

$$
\lim _{x \rightarrow 0} f^{\prime}(x) \exp (-f(x / 2) / \alpha)=0,
$$

5) for some $\beta>1, \beta f(x) \leqslant f(x / 2)$.

This completes the list of our assumptions. 
Comments on the assumptions. Note that the fifth postulate implies that $f$ tends to infinity at 0

The assumptions are rather technical. It might be a good idea to have in mind the singularity

$$
x \rightarrow \operatorname{sgn}(x) \exp (-1 /|x|) .
$$

In fact, the abstract hypotheses were developed with the objective to catch essential properties of this singularity.

THE MAIN THEOREM. Under the hypotheses listed above all orbits of $h(\bmod 1)$ are dense on the circle.

1.4. Strategy of the proof. We will follow the ideas of [3]. In order to show that the orbits are dense it is sufficient to demonstrate that there are no wandering intervals. We assume that there is one and try to show that the line of argument used in [3] to disprove that still works in our situation. If $h$ satisfied the cross-ratio inequality on the whole circle that would be easy, but since the singularity is not quasisymmetric, the cross-ratio inequality cannot hold. For example, the cross-ratio may grow by an arbitrary factor if $a<b<0<c<d$ and all are close to 0 . Nevertheless, our assumptions imply a very strong decrease of the cross-ratio if all four points are on one side of 0 in $U$. We intend to show that this decrease is sufficient to offset the possibly unbounded growth. The main difficulty in carrying out this kind of estimate is that the amount by which a cross-ratio is decreased is of the second order with respect to the length of $(a, d)$. Thus, we will have to show that the images of the alleged wandering interval have a definite size in a close neighborhood of the singularity.

\section{Proof}

\subsection{A few estimates}

LEMMA 2.1. Take points $a, b, c$ in $U$ that satisfy $0<c<b<a$ and $f(c)-f(b) \leqslant 1000$. The inequality

$$
\frac{|h(a)-h(b)|}{|h(a)-h(c)|} \cdot \frac{|a-c|}{|b-a|} \leqslant K_{2,1}
$$

then holds where $K_{2.1}$ is a constant that depends on $h$ only.

Proof. We compute

$$
\frac{|h(a)-h(b)|}{|h(a)-h(c)|} \cdot \frac{|a-c|}{|b-a|}=\frac{|\exp (f(a)-f(b))-1|}{|\exp (f(a)-f(c))-1|} \cdot \frac{|a-c|}{|b-a|} .
$$

First, we consider the case when $f(b)-f^{\prime}(a)>f(c)-f^{\prime}(b)$. As $f^{\prime \prime}$ was assumed to be positive, that implies $b-a>c-b$ and $K_{2.1}=2$ is enough to satisfy the inequality in this case. Thus, we further assume that $f(b)-f(a) \leqslant f(c)-f^{\prime}(b)$, which in particular implies that $f(c)-f(a) \leqslant 2000$. Then

$$
1-\exp (f(a)-f(c))>\frac{1-\exp (-2000)}{2000}(f(c)-f(a))
$$

We only have to substitute estimate (2) and the obvious inequality

$$
1-\exp (f(a)-f(b))<f(b)-f(a)
$$

into equation (1) to conclude the proof.

LEMMA 2.2. Let $0<a<b$ and both $a$ and $b$ belong to $U$. Suppose also that $f(a)-f(b)>10$. Then, if $|a|,|b|<K_{2,2,1}$, the inequality

$$
\log \left(\frac{h^{\prime}(a) h^{\prime}(b)}{(h(a)-h(b))^{2}}(b-a)^{2}\right) \leqslant K_{2.2,2}(f(b)-f(a))
$$

holds where $K_{2,2,1}$ and $K_{2,2,2}$ are positive constants that only depend on $h\left({ }^{2}\right)$.

Before we prove that lemma, we want to emphasize that the argument of the logarithm on the left-hand side of the inequality is essentially the same as the quantity used in [3].

Proof. We compute

$$
\log \left(\frac{h^{\prime}(a) h^{\prime}(b)}{(h(a)-h(b))^{2}}(b-a)^{2}\right)=\frac{f^{\prime}(a) f^{\prime}(b) \exp (f(a)-f(b))}{(\exp (f(a)-f(b))-1)^{2}}(b-a)^{2} .
$$

Since $f(a)-f(b)>10$, it follows that

$$
\exp (f(a)-f(b))-1>0.5 \exp (f(a)-f(b)) .
$$

By the Mean Value Theorem we can find a point $\zeta \in(a, b)$ which satisfies $f^{\prime}(\zeta)(b-a)$ $=f(b)-f(a)$. Consequently, the logarithm of equation (3) does not exceed

(4) $\log 4+f(b)-f(a)+\log \left|f^{\prime}(a)\right|+\log \left|f^{\prime}(b)\right|+2 \log (b-a)$

$$
=\log 4+f(b)-f(a)+2 \log |f(b)-f(a)|-\log \left|f^{\prime}(\zeta)\right|+\log \left|f^{\prime}(b)\right|-\log \left|f^{\prime}(\zeta)\right| .
$$

Expression (4) can be further estimated from above by

(5) $\quad 2(f(b)-f(a))+\log \left|f^{\prime}(a)\right|-\log \left|f^{\prime}(\zeta)\right|+\log \left|f^{\prime}(b)\right|-\log \left|f^{\prime}(\zeta)\right|$

$$
=2(f(b)-f(a))+\left|\left(\log f^{\prime}\right)^{\prime}(\xi)\right|+\left|\left(\log f^{\prime}\right)^{\prime}\left(\xi^{\prime}\right)\right| .
$$

To get this estimate of (4) we have used the inequality $\log |x| \leqslant x / e$. The points $\xi$ and $\xi^{\prime}$ both belong to $(a, b)$.

Let us assume for a moment that $b / a<2$. Then, item 3 in our hypotheses implies that provided $b$ is close enough to 0 , the $2(f(b)-f(a))$ term dominates in the right-hand side of equation (5) and Lemma 2.2 follows.

The general situation can be reduced to this case if we prove that for any $c$ between $a$ and $b$

(6) $\log \left(\frac{h^{\prime}(a) h^{\prime}(b)}{(h(a)-h(b))^{2}}(b-a)^{2}\right) \leqslant \log \left(\frac{h^{\prime}(a) h^{\prime}(c)}{(h(a)-h(c))^{2}}(c-a)^{2}\right)+\log \left(\frac{h^{\prime}(c) h^{\prime}(b)}{(h(b)-h(c))^{2}}(b-c)^{2}\right)$.

$\left({ }^{2}\right)$ Note that the right-hand side of the estimate is negative. 
However, inequality (6) is an immediate corollary to the fact that $h$ decreases all cross-ratios in $U$. The difference between the left-hand side and the right-hand side in this inequality equals

$$
2 \log \frac{\frac{(h(b)-h(c))(h(c)-h(a))}{(b-c)(c-a)}}{\frac{h(b)-h(a)}{b-a} h^{\prime}(c)} .
$$

The argument of this logarithm is the distortion of some cross-ratio under $h$. It is, therefore, less than 1.

From now on, $U$ will denote a smaller neighborhood of 0 , namely $B\left(0, K_{2,2,1}\right)$. LEMMA 2.3. Take points $a$ and $b$ both in $U$. Assume that $a<0<b$ and $|b| \geqslant|a|$ as well. Then

$$
\frac{h^{\prime}(a) h^{\prime}(b)(b+a)^{2}}{(h(b)-h(a))^{2}} \leqslant 4\left(\frac{h^{\prime}(b) b}{h(b)-h(0)}\right)^{2} .
$$

Proof. Since $h(b)-h(0) \leqslant \frac{1}{2}(h(b)-h(a))$ and $h^{\prime}(a)<h^{\prime}(b)$, the lemma follows.

2.2. Basic Lemma. We suppose that there is a wandering interval $I$.

DefinItION 2.1. A pair of positive integers $(m, n)$ will be called a critical pair if it satisfies the conditions listed below:

1) the intervals $h^{m}(I)$ and $h^{n}(I)$ are both contained in $U$,

2) the point 0 is between $h^{m}(I)$ and $h^{n}(I)$,

3) for no other $i<\max (m, n)$ does the $i$ th image of $I$ fall between $h^{n}(I)$ and $h^{m}(I)$.

Properties of critical pairs. We give three simple lemmas which explain the dynamical role played by critical pairs.

DefinItION 2.2. For a degree one circle homeomorphism with an irrational rotation number equal to $\varrho$ we define a sequence of positive integers $q_{i}$ called the closest returns of the map. The zeroth closest return, $q_{0}$, is always 1 . The $k$ th closest return is inductively defined to be the smallest integer $i$ greater than $q_{k-1}$ for which

$$
\operatorname{dist}(j \varrho, Z)<\operatorname{dist}\left(q_{k-1} \varrho, Z\right)\left({ }^{3}\right) \text {. }
$$

LemMa 2.4. Denote by $l$ the lowest iterate of $h$ which maps $I$ into the interval between $h^{m}(I)$ and $h^{n}(I)$. Then at least one of the numbers $l-m$ and $l-n$ is a closest return.

Proof. It is well-known that $h$ and the rigid rotation by the rotation number of $h$ are topologically semi-conjugate. This means that there is a continuous map $G: S^{1} \rightarrow S^{1}$ that satisfies $G \circ h=R_{e} \circ G$. Furthermore, $G$ is weakly order preserving and squeezes $I$ into a point. Since in our situation $G\left(h^{n}(I)\right)$ and $G\left(h^{m}(I)\right)$ are the closest neighbors of $G\left(h^{l}(I)\right)$, one at least must be a closest return of $R_{-\varrho}$.

${ }^{(3)}$ Therefore, the closest returns only depend on the rotation number.
Notational conventions. In the following two lemmas, we keep the notations from Lemma 2.4. In addition, we assume that $h^{n}(I)$ is on the right of 0 and $l=n+q_{k}$. It is literally so if $k$ is an odd number; otherwise if we want to preserve the relation $l=n+q_{k}$, then $h^{n}(I)$ is on the left of 0 . However, since the analytic assumptions we made about $h$ are insensitive to a change of the orientation, without loss of generality we may only consider the situation with $h^{n}(I)$ on the right side of 0

We denote the endpoints of $I$ as follows: $I=(y, x)$.

Two more lemmas. Let $\mathscr{I}$ denote the set of all positive integers $i$ for which $f^{i}(I)$ falls between $h^{m}(I)$ and $h^{n}(I)$.

LEMMA 2.5. If $s \in \mathscr{I}$, then the next larger number in $\mathscr{I}$ is $s+q_{k}$.

Proof. Suppose $s+j \in \mathscr{I}$ and $0<j<q_{k}$. We choose $s$ and $j$ so as to satisfy this with the smallest possible. If $h^{s+j}(I)$ were on the right of $h^{s}(I)$, then $n+j<l$ would be in $\mathscr{I}$, contrary to the definition of $l$. Otherwise, $m+j \in \mathscr{I}$ and we arrive at the same contradiction, unless $m>n$. In that case, we can set $s=n+q_{k}$ and $j^{\prime}=m+j-n-q_{k}$. As $m<n+q_{k}$, this new $j^{\prime}$ is less than $j$, contrary to the way the pair $(s, j)$ was chosen.

LEMMA 2.6. If $s<q_{k+1}$ and $s \in \mathscr{I}$, then $s+q_{k} \in \mathscr{I}$. Let

$$
T:=\min \left\{s \in \mathscr{I}: s+q_{k} \notin \mathscr{I}\right\} .
$$

Then $T=m+q_{k+1}$.

Proof. If $s$ is in $\mathscr{I}$ but $s+q_{k}$ is not, then $h^{m}(I)$ is contained in $\left(h^{s+q_{k}}(x), h^{s}(y)\right)$. Because of the properties of the closest returns, the first time the backward orbit of $h^{s}$ visits this interval is after $-q_{k+1}$ iterates. Hence, $m \leqslant s-q_{k+, 1}$ and Lemma 2.6 follows.

The situation is pictured in Figure 1 below. Starting from $n$, each $q_{k}$ iterates move the image of $I$ to the left towards $h^{m}(I)$. The last one which is still between $h^{m}(I)$ and $h^{n}(I)$ is $h^{T}(I)$. The number $t$ is chosen to be the largest in $\mathscr{I}$ for which the corresponding image of $I$ is still on the right of 0 .

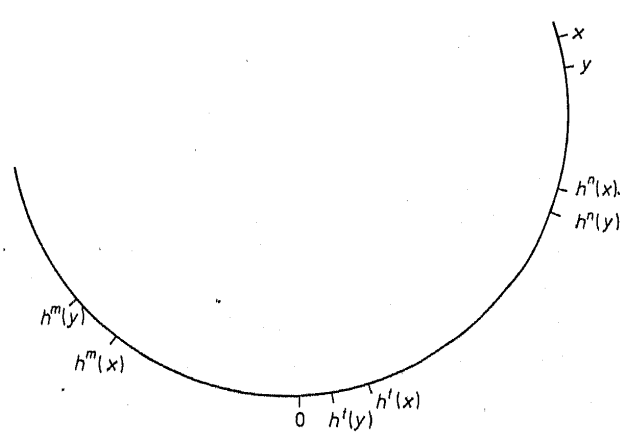

Fig. 1 
Some more notations. For every critical pair $P=(m, n)$ we define two numbers:

$$
\begin{gathered}
u(P):=\operatorname{dist}\left(0, h^{n}(I) \cup h^{m}(I)\right), \\
v(P):=\min \left\{\sup \left\{\operatorname{dist}(0, x): x \in h^{j}(I)\right\}: j=m, n\right\} .
\end{gathered}
$$

That is, $v(P)$ is the distance from 0 to the closest of the farther endpoints of $h^{n}(I)$ and $h^{m}(I)$.

BASIC Lemma. Consider a critical pair $P=(m, n)$. If $\min (m, n)$ is sufficiently large, then, with a suitable choice of a positive constant $B_{1}$ independent of $P$, another critical pair $P^{\prime}=\left(m^{\prime}, n^{\prime}\right)$ can be found so as satisfy these three conditions:

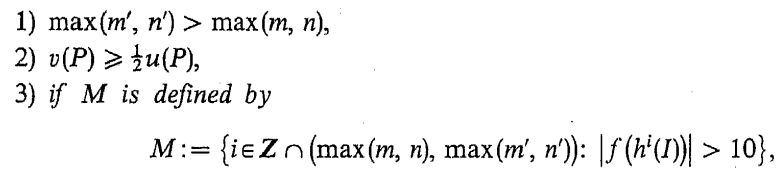

then

$$
\sum_{i \in M}\left|f\left(h^{i}(I)\right)\right| \geqslant B_{1}\left(f\left(u\left(P^{\prime}\right)\right)-f(u(P))\right) .
$$

Beginning of the proof. The proof of the Basic Lemma is fairly complicated and involves a couple of cases to be considered separately. The first split depends on whether $\left|h^{T-q_{k}}(x)\right|>\left|h^{m}(x)\right|$ or not. If this inequality holds true, we set $m^{\prime}=m$ and $n^{\prime}=T-q_{k}$. In particular, the inequality implies that $h^{n^{\prime}}(I)$ is on the right side of 0 and, therfore, $\left(m^{\prime}, n^{\prime}\right)$ is a critical pair. The hard part in verifying the three conditions of the Basic Lemma is to understand them: they are all trivially true.

So in the remaining case we may assume

$$
\left|h^{m}(x)\right| \geqslant\left|h^{T-q_{k}}(x)\right| \text {. }
$$

The advantage of this additional assumption is that the next lemma is then true.

Lemma 2.7. Pick points $z$ and $z^{\prime}$ in such a way that $z$ is in the interval $\left(x, h^{q_{k+1}-y_{k}}(x)\right)$ and $z^{\prime}$ belongs to $\left[h^{q_{k}-q_{k+1}}(y), y\right)$. If $\left|h^{t+q_{k}}(x)\right|<\left|h^{t}(y)\right|$ and with our additional assumption, the estimate

$$
D\left(z^{\prime}, y, x, z ; h^{j}\right) \leqslant 8 C_{5} D\left(h^{n}\left(z^{\prime}\right), h^{n}(y), h^{n}(x), h^{n}(z) ; h\right)
$$

holds for every $j \leqslant \max (m, n)\left({ }^{4}\right)$.

Proof. The cross-ratio inequality guarantees that the possible increase of the cross-ratio under all iterates $i$ for which $\left(h^{i-1}\left(z^{\prime}\right), h^{i-1}(z)\right) \nexists 0$ is bounded by $C_{5}$. The effect of the $n$th iterate is factored in explicitly; so we only have to show that the joint increase caused by all other iterates can be bounded by 8 .

We look for the iterates $i$ by which the image of $\left(z^{\prime}, z\right)$ covers 0 and the image of $I$ falls to the left of 0 . Then, either $i=m$ or $h^{i}(I)$ is further from 0 than $h^{m}(0)$. Note that

$\left({ }^{4}\right)$ The constant $C_{5}$ comes from the cross-ratio inequality. the cross-ratio

$$
\operatorname{Cr}\left(h^{i}\left(z^{\prime}\right), h^{i}(y), h^{i}(x), 0\right)
$$

will be decreased under the next iterate of the map. Because of our additional assumption, the resulting cross-ratio is at least a half of

$$
\operatorname{Cr}\left(h^{i+1}\left(z^{\prime}\right), h^{i+1}(y), h^{i+1}(x), h^{i+1}(z)\right) \text {. }
$$

So, the $(i+1)$ st iterate cannot increase the cross-ratio by more than 2 . Also, the way $z$ and $z^{\prime}$ were chosen guarantees that this situation happens at most twice. Thus, so far we have seen growth by a factor of at most 4 .

Next, we consider the situation when the $h^{i}\left(z^{\prime}, z\right)$ covers 0 and $h^{i}(I)$ is on the right. Again, we see that there is at most one such $i$ other than $n$. Moreover, in this case $h^{i}(I)$ is on the right of $h^{n}(I)$. Hence, we come to the conclusion that if $t>n$, there is $i$ other than $n$ for which $f^{i}\left(z^{\prime}, z\right)$ covers 0 and $f^{i}(I)$ is on the right of 0 . If $t=n$, then our assumption comes into play, which implies that $\left|h^{t}\left(z^{\prime}\right)\right|<\left|h^{t}(y)\right|$. The reasoning that we used in the previous case works again and, therefore, we are able to show that the cross-ratio grows at most twice.

Why we will not verify the assumptions of Lemma 2.7 in the future. The lemma has two important assumptions and we already argued that one of them can always be adopted without loss of generality. The other is that $\left|h^{t+q_{k}}(x)\right|<\left|h^{t}(y)\right|$.

We note that when this inequality is not satisfied it is very easy to satisfy the claim of the Basic Lemma. We pick $m^{\prime}=t$ and $n^{\prime}=t+q_{k}$ and again we straightforwardly check that this is a good choice.

In fact, this choice of $P^{\prime}$ is going to work in all remaining cases as well, but the proof will no longer be easy. Before we can tackle it, we need more technical lemmas.

So far, we have established that unless we can immediately conclude the proof of the Basic Lemma, Lemma 2.7 holds.

More lemmas. We prove two more lemmas, in which we implicitly make the same assumptions as in Lemma 2.7

LEMMA 2.8. Let $j$ be the least integer with the following property: either $t+j q_{k}>T$ or $\left|h^{t+j q_{k}}(y)\right|>\left|h^{t}(x)\right|$. Then

and

$$
\left|h^{t+j q_{k}}(y)\right| /\left|h^{l+q_{k}}(y)\right|<2
$$

$$
\left|f\left(h^{t+q_{k}}(I)\right)\right|>K_{2,8}\left|f\left(h^{t+q_{k}}(x)\right)-f\left(h^{t+j q_{k}}(x)\right)\right|
$$

where $K_{2,8}$ is positive and depends on $h$ only.

Proof. If $\left|h^{t+q_{k}}(y)\right|>\left|h^{t}(x)\right|$, there is nothing to prove. Hence, we assume that the reverse inequality is true. By Lemma 2.7 , we can make

$$
\operatorname{Cr}\left(h^{t+q_{k}}(y), h^{t}(y), h^{t}(x), h^{t-q_{k}}(x)\right)
$$

arbitrarily small provided $k$ is large enough, that is, $\min (m, n)$ is sufficiently big. Let $\chi$ 
denote the value of this cross-ratio. It certainly may grow after another iterate, but we can bound its growth $\left(^{5}\right)$. Namely, we substitute $h^{t+q_{k}}(y)$ with 0 in the quadruple, and study how the new cross-ratio changes under the next iterate. Obviously, its value will drop, and since $f$ is even, the resulting cross-ratio will be at least half as large as $\operatorname{Cr}\left(h^{t+q_{k}+1}(y), h^{t+1}(y), h^{t+1}(x), h^{t-q_{k}+1}(x)\right)$. Therefore,

$$
\operatorname{Cr}\left(h^{t+2 q_{k}}(y), h^{t+q_{k}}(y), h^{t+q_{k}}(x), h^{t}(x)\right)<2 \chi .
$$

On the other hand, $\left|\left(h^{t+q_{k}}(x), h^{t}(x)\right)\right| /\left|\left(h^{t+q_{k}}(y), h^{t}(x)\right)\right|>1 / 2$. Thus,

$$
\left|\left(h^{t+2 q_{k}}(y), h^{t+q_{k}}(y)\right)\right| /\left|\left(h^{t+q_{k}}(y), h^{t+q_{k}}(x)\right)\right|<5 \chi \text {. }
$$

If $j$ is 2, Lemma 2.8 already follows. Otherwise, we have to repeat the argument. This time, we begin with the quadruple $h^{t+2 q_{k}}(y), h^{t}(y), h^{t}(x), h^{t-q_{k}}(x)$. We have shown that their cross-ratio does not exceed $\chi(1+5 \chi)$.

This procedure can be repeated until we reach $j$. Set

$$
c_{i}:=\left|\left(h^{t+i q_{k}}(y), h^{t+q_{k}}(x)\right)\right| \text {. }
$$

Then our reasoning proves that the recursive condition

$$
c_{i}<c_{1}\left(1+5 \chi c_{i-1} / c_{1}\right)
$$

is satisfied provided $i \leqslant j$.

Next, it is an elementary observation that the sequence $c_{i}$ is bounded by $c_{1} /(1-5 \chi)$ Since, as we noted at the beginning of the proof, we may assume that $\chi$ is small, it follows that the sequence $c_{i}$ is bounded depending on $h$ only. This immediately implies the lemma if we also remember that $f$, was assumed to be convex.

LEMMA 2.9. Suppose that $\left(w_{i}\right)_{i \in J}$ is a growing sequence of positive numbers that are all in $U$. We are also given another similar sequence $\left(W_{i}\right)_{i \in J}$ which also satisfies $W_{i} \in\left(w_{i}, w_{i+1}\right)$ for all $i \in J$. Furthermore,

$$
\frac{W_{i}-w_{i}}{W_{i+1}-W_{i}}>100
$$

for every $i \in J$. Then:

- If $\mu$ is less than $w_{i}$ for some $i \in J$, the ratio $\mu / W_{1}$ is less than 2 .

- If, in addition, $f\left(w_{1}\right)-f(\mu) \geqslant 100$ and $J^{\prime}$ denotes the set

then

$$
J^{\prime}:=\left\{i \in J: W_{i}<\mu \text { and }\left|f\left(w_{l}, W_{i}\right)\right|>10\right\}
$$

$$
\sum_{i \in J^{\prime}}\left|f\left(w_{i}, W_{i}\right)\right|>K_{2.9}\left(f\left(w_{1}\right)-f(\mu)\right)
$$

with a positive constant $K_{2.9}$ that depends on $h$ only.

Proof. The lemma can be proved straightforwardly by calculations that use the rules for summing geometric series and the fact that $f$ is a convex function. We omit this argument.

$\left({ }^{5}\right)$ We used an analogous trick in the proof of Lemma 2.7 .
The proof of the Basic Lemma continued. Armed with these lemmas we are ready to consider the remaining cases and finally prove the Basic Lemma. We always assume that we are not in one of the situations that have already been dealt with.

Case A: $\left|h^{t+q_{k}}(y)-h^{t}(y)\right| /\left|h^{t}(y)-h^{t}(x)\right|<1 / 3$. We distinguish two subcases.

Subcase A1: $\left|h^{t+q_{k}}(x)\right| \geqslant\left|h^{t}(y)\right|$. Then the cross-ratio

$$
\operatorname{Cr}\left(h^{t+1+q_{k}}(y), h^{t+1}(y), h^{t+1}(x), h^{t-q_{k}+1}(y)\right)
$$

is very small; in fact, it can be as small as we want provided we have chosen $U$ to be narrow enough. The assumption of the present subcase implies that the distortion of this cross-ratio by the subsequent $T-t-1$ iterates is bounded by a uniform constant. Hence, by picking a small $U$, we may ensure that the lengths of the intervals $\left(h^{t+j q_{k}}(y), h^{t+(j-1) q_{k}}(y)\right)$ decay exponentially with $j$ until $t+j q_{k}>q_{k+1}$ with the ratio which is less than 0.01 . Since the last of these intervals contains $h^{m}(I)$, we have

$$
\left|h^{m}(x)\right| /\left|h^{t}(y)\right|<2
$$

which proves property 2 claimed in the Basic Lemma. Property 3 is even more obvious, since $\left|h^{t}(x)\right|>\left|h^{m}(x)\right|$ and thus $\left|f\left(h^{t}(I)\right)\right|$ alone is greater than $f\left(u\left(\mathscr{P}^{\prime}\right)\right)-f(u(\mathscr{P}))$.

This concludes the proof in subcase A1.

Subcase A2: $\left|h^{t}(y)\right|>\left|h^{t+q_{k}}(x)\right|$. This case is immediately solved using Lemma 2.8 .

Thus we have successfully dealt with Case A.

Case B: $\left|\left(h^{t+q_{k}}(y), h^{t}(y)\right)\right| /\left|\left(h^{t}(y), h^{t}(x)\right)\right| \geqslant 1 / 3$. Since the cross-ratio

$$
\operatorname{Cr}\left(h^{t+q_{k}}(y), h^{t}(y), h^{t}(x), h^{t-q_{k}}(x)\right)
$$

is small if $\min (m, n)$ is sufficiently large, the ratio $\left|\left(h^{t}(x), h^{t-q_{k}}(x)\right)\right| /\left|h^{t}(y), h^{t}(x)\right|$ is very small. If $t-q_{k} \geqslant n$, we also know that the cross-ratio

$$
\operatorname{Cr}\left(h^{t}(y), h^{t-q_{k}}(y), h^{t-q_{k}}(x), h^{t-2 q_{k}}(x)\right)
$$

is small. By the same reasoning, we can verify that if we substitute $w_{i}:=h^{t-q_{k}}(y)$ and $W_{i}:=h^{t-i l_{k}}(x)$, we are in the situation of Lemma 2.9.

If $\left|h^{t}(y)\right| \leqslant\left|h^{t+q_{k}}(x)\right|$ and $\left|h^{t}(I)\right|>100$, then Lemma 2.9 implies the properties claimed in the Basic Lemma. Next, we show that if $\left|f\left(h^{t}(I)\right)\right| \leqslant 10$, then $\left|h^{t+q_{k}}(x)\right|<\left|h^{t}(y)\right|$ and $\left|f^{\prime}\left(h^{t+q_{k}}(I)\right)\right|>100$.

To this end, consider the quadruple $h^{q_{k}-q_{k+1}}(y), y, x, h^{q_{k+1}-q_{k}}(x)$. We look at the $q_{k+1}-1$ consecutive images of these points and their cross-ratios. An important observation is that not all of those cross-ratios are small; one at least is not less than a quarter. To see that this must be the case, choose $j \in\left[0, q_{k+1}-1\right]$ with the smallest length of $h^{J}(I)$. It is an easy property of the dynamics of the irrational rotation that both $h^{j}\left(h^{q_{k}-q_{k+1}+1}(y), y\right)$ and $h^{J}\left(x, h^{q_{k+1}-q_{k}}(x)\right)$ contain another image of $I$ by an iterate earlier than $q_{k+1}$.

On the other hand, we know that originally this cross-ratio is very small, provided $\min (m, n)$ is large. By Lemma 2.7, it remains small at least for $i \leqslant n$. After that, an increase by more than a constant factor is only possible when $h^{i}\left(h^{q_{k}-q_{k+1}}(y), h^{q_{k+1}-q_{k}}(x)\right)$ 
contains 0 . If $h^{i}(I)$ is on the right of 0 , the increase is bounded by Lemma 2.1. The assumptions of the Lemma are satisfied, because we explicitly assumed $\left|f\left(h^{t}(y), h^{t}(x)\right)\right| \leqslant 100$ and $\left|f\left(h^{t}(x), h^{t+q_{k+1}-q_{k}}(x)\right)\right|$ is yet much less as

$$
\operatorname{Cr}\left(h^{t+q_{k}-q_{k+1}}(y), h^{t}(y), h^{\prime}(x), h^{t+q_{k+1}-q_{k}}(x)\right)
$$

is small.

Let us assume that $\left|h^{t+q_{k}}(x)\right| \geqslant\left|h^{t}(y)\right|$ and we will show that this leads to a contradiction. Namely, if this inequality held true, the cross-ratio could only grow by a bounded factor. Indeed, only the case when $h^{\prime}(I)$ is on the left side of 0 and $h^{i}\left(h^{q_{k}-q_{k+1}}(y), h^{q_{k+1}-q_{k}}(x)\right) \ni 0$ is left to be considered. We replace $h^{q_{k+1}-q_{k}}(x)$ by () and fix our attention on the quadruple

$$
h^{q_{k}-q_{k+1}+i}(y), h^{i}(y), h^{i}(x), 0
$$

and its cross-ratio. It will be decreased by the next iterate. If $\left|h^{t+1 / g_{k}}(. x)\right| \geqslant\left|h^{q_{k+1}-q_{k}}(x)\right|$, that would imply that

$$
D\left(h^{q_{k}-q_{k+1}+i}(y), h^{i}(y), h^{i}(x), h^{q_{k+1}-q_{k}+i}(x) ; h\right) \leqslant 2 .
$$

In fact, we only know that $\left|h^{t+q_{k}}(x)\right| \geqslant\left|h^{t}(y)\right|$. However, $f^{\prime}\left(h^{t}(y)\right) \cdots \cdot f\left(h^{q_{k+1}-y_{k}}(x)\right)$ $\leqslant 1.01 f\left(h^{t}(I)\right)$ provided $\min (m, n)$ is large. Since our assumption was that $f\left(h^{t}(I)\right)<100$, it follows that $f\left(h^{t}(y)\right)-f\left(h^{q_{k+1}-q_{k}}(x)\right) \leqslant 101$, which only contributes another bounded factor of $\exp (101)$ to our estimate.

Therefore, we have proved that $\left|h^{\ell^{+}+q_{k}}(x)\right|<\left|h^{t}(y)\right|$. In a similar way we can argue that $\left|f\left(h^{t+q_{k}}(I)\right)\right|>100$. Otherwise, we prove that the cross-ratio can grow only by a bounded factor by repeating the argument we used in the previous case when $h^{i}(I)$ was on the right of 0 .

The conclusion of proof. The reader may check that in all cases not yet dealt with in the previous subsection, $\left|h^{t+q_{k}}(x)\right|<\left|h^{t}(y)\right|$, and if $\left|f\left(h^{t+q_{k}}(I)\right)\right|<100$, then $\left|f^{\prime}\left(h^{t}(I)\right)\right| \geqslant 100$. Then Lemmas 2.8 and 2.9 enable us to immediately conclude the proof.

We have completed the proof of the Basic Lemma.

COROLlary. The Basic: Lemma implies that we can construst an infinite sequence of critical pairs. They descend to 0 and the claim of the Busic Lemma holds for any two consecutive pairs in the sequence. We will call this sequence the critical sequence.

2.3. Proof of the main theorem. The Basic Lemma and the existence of the critical sequence let us quickly conclude the proof of our main theorem.

We will trace the lines of Yoccoz' proof (sec [3]). Accordingly, we consider $h^{-q_{r+1}}(I), I, h^{-q_{r+1}-q_{r}}(I)$ for a large $r$. We also find points $X$ and $Y$ which satisfy

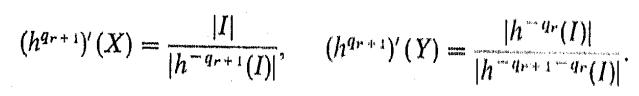

As was shown in [3], the distortion of the infinitesimal cross-ratio defined by these points tends to infinity with $r$. On the other hand, the cross-ratio inequality implies that there is only one iterate of $h$ under which the cross-ratio is increased: namely the, say, $(L+1)$ st iterate where $h^{L}(X, Y)$ contains 0 .

Next, we consider two consecutive pairs from the critical sequence. Those are the last pair for which $h^{L}(X, Y)$ is contained between the intervals of the pair, and the next pair from the critical sequence. We denote the earlier pair with $P=(m, n)$ and the later one with $P^{\prime}=\left(m^{\prime}, n^{\prime}\right)$ and use the Basic Lemma.

By Lemma 2.3 the logarithm of the factor by which the $(L+1)$ st iterate increases the cross-ratio is bounded by $2 \log (u(P))\left|f^{\prime}\left(v\left(P^{\prime}\right)\right)\right|$. At the same time, the Basic Lemma allows us to estimate the logarithmic decay of the cross-ratio under all other iterates. That is proportional to $f(u(P))$. According to the technical assumption 4 in the statement of the main theorem, the second effect will prevail for large $r$. Therefore, the cross-ratio cannot grow as it would have to, and we have arrived at a contradiction.

Thus, there is no wandering interval and the proof is finished.

\section{References}

[1] R. Hall, $A C^{\infty}$ Denjoy counterexample, Ergodic Theory Dynamical Systems 1 (1981), 261-272. 2] G. S wi ą tek, Rational rotation numbers for maps of the circle, Comm. Math. Phys. 119 (1988), 109-128. [3] J.-C. Yocco z, Il n'y a pas de contre-exemple de Denjoy analytique, C.R. Acad. Sci. Paris 298 (7)
(1984), 141-144.

\section{INSTITUTE FOR MATHEMATICAL SCIENCES}

SUNY AT STONY BROOK

Stony Brook, L.I., New York 11794

Received 13 June 1989;

in revised form 12 March 1990 and 22 August 1990 